\title{
MicroRNA Dysregulation in the Hippocampus of Rats with Noise- Induced Hearing Loss
}

\author{
Seungmin Ha, Kyung Woon Kim, So Min Lee, Chang Ho Lee, and So Young Kim \\ Department of Otorhinolaryngology Head \& Neck Surgery, CHA University College of Medicine, \\ Bundang CHA Medical Center, Republic of Korea \\ Correspondence should be addressed to So Young Kim; sossi81@hanmail.net
}

Received 8 April 2021; Revised 17 August 2021; Accepted 20 August 2021; Published 6 September 2021

Academic Editor: Nadja Schroder

Copyright ( 2021 Seungmin Ha et al. This is an open access article distributed under the Creative Commons Attribution License, which permits unrestricted use, distribution, and reproduction in any medium, provided the original work is properly cited.

\begin{abstract}
Although hippocampal changes due to noise-induced hearing loss have been suggested, little is known about the miRNA levels due to these hippocampal changes. Three-week-old Sprague-Dawley rats were divided into noise and control groups $(n=20$ per group). The noise group rats were exposed to white Gaussian noise (115 dB SPL, 4 hours per day) for three days. One day after noise exposure, the hippocampi of rats were harvested and miRNA expressions were analyzed using the Affymetrix miRNA 4.0 microarray $(n=6$ per group). The predicted target genes of each miRNA were retrieved, and the pathways related to the predicted target genes were analyzed. miR-758-5p, miR-210-5p, miR-370-5p, miR-652-5p, miR-3544, miR-128-1-5p, miR-665, miR-188-5p, and miR-874-5p expression increased in the hippocampal tissue of the noise group compared to that in the control group. The overlapping predicted target genes included Bend4, Creb1, Adcy6, Creb5, Kcnj9, and Pten. The pathways related to these genes were the estrogen signaling pathway, vasopressin-regulated water reabsorption, thyroid hormone synthesis, aldosterone synthesis and secretion, insulin secretion, circadian entrainment, insulin resistance, cholinergic synapse, dopaminergic synapse, cGMP-PKG signaling pathway, cAMP signaling pathway, PI3K-Akt signaling pathway, TNF signaling pathway, and AMPK signaling pathway. miR-448-3p, miR204-5p, and miR-204-3p expression decreased in the hippocampal tissue of the noise group compared to that in the control group. The overlapping predicted target genes of these three miRNAs were Rps6kas, Nfactc3, Rictor, Spred1, Cdh4, $C d h 6, D v l 3$, and Rcyt 1b. Pathway analysis suggested that the Wnt signaling pathway is related to Dvl3 and Nfactc3. Noise-induced hearing loss dysregulates miR-758-5p, miR210-5p, miR370-5p, miR-652-5p, miR-3544, miR-128-1-5p, miR-665, miR-188-5p, miR-874-5p, miR-448-3p, miR-204-5p, miR-204-3p, and miR-140-5p expression in the hippocampus. These miRNAs have been predicted to be associated with hormonal, inflammatory, and synaptic pathways.
\end{abstract}

\section{Introduction}

Hearing loss affects daily life in many aspects; in particular, it hampers communication and causes psychosocial problems such as social isolation and depression [1]. One of the main causes of this handicapped condition is exposure to extreme noises [2]. Such noise exposure negatively affects hair cells in the cochlea and neurons in the auditory pathway.

Hearing loss often accompanies impaired communication skills and social isolation, which may lead to cognitive decline. The hippocampal region of the brain is involved in learning and memory, and several studies have supported the hypothesis that hearing loss induces cognitive deficit [3].
Our previous microarray study showed that acute noise exposure alters hippocampal gene expression [4]. Another study on an Alzheimer's disease (AD) model has reported that hearing loss induces cognitive deficits and reduced synapse in the hippocampus [5]. Moreover, the expression of Wnt signaling pathway molecules reduced, and amyloid- $\beta$ and tau accumulated in the hippocampi of mice after chronic noise exposure [6].

MicroRNAs (miRNAs) are small, noncoding, singlestranded RNAs that inactivate messenger RNAs (mRNAs) [7]. miRNAs posttranscriptionally regulate gene expression almost throughout the entire human genome [8]. Since miRNAs are more stable than proteins after repeated freeze-thaw 
cycles, several studies have attempted to develop new biomarkers or therapeutics using miRNAs [9]. In addition, recent studies have shown a relationship between cognitive decline and miRNAs in $\mathrm{AD}$. In a rat model of $\mathrm{AD}$, several miRNAs were found to be involved in cognitive deficits after hearing loss [10]. Another study using aged Tg4-42 mice, a sporadic $\mathrm{AD}$ model revealed alterations in hippocampal miRNAs after memory decline [11].

Previous studies on miRNAs have mostly focused on chronic noise-induced hearing loss models. However, a previous study measured miRNA expression in the cochlear nucleus and inferior colliculus, not the hippocampus, after acute noise exposure [12]. Since noise-induced hearing loss and related cognitive decline may progress slowly over a long period, early diagnosis and prevention are important. Therefore, we aimed to examine the changes in hippocampal miRNAs, particularly in an early hearing loss model.

\section{Materials and Methods}

2.1. Animal Groups and Noise Exposure. This study was approved by the Institutional Animal Care and Use Committee of the CHA University Medical School (IACUC190046). The conditions for raising animals, noise exposure, anesthesia, and sacrifice procedures conformed to the regulations of the Institutional Animal Care and Use Committee of CHA University Medical School. Threeweek-old Sprague-Dawley rats were divided into control and noise groups (Figure 1). The rats in the noise group were exposed to $2-20 \mathrm{kHz}, 115 \mathrm{~dB}$ SPL noise ( $4 \mathrm{~h} /$ day for 3 days) via a free-field speaker (Tucker-Davis Technologies; Alachua, FL, USA) implemented at the top of the noise box. The noise was exposed intermittently for 3 days to attenuate stress response from continuous traumatic noise stimulation, which was lethal for some rats. Under this noise exposure schedule, hearing thresholds were not recovered over 3 months after cessation of noise exposure. The control group rats were exposed to 40-60 dB SPL background noise for $4 \mathrm{~h} /$ day for 3 days. All rats were not anesthetized during noise exposure. The auditory brainstem response (ABR) thresholds were measured before and after noise exposure. One day after noise exposure, all rats were euthanized and the dorsal cornu ammonis 3 region of the hippocampi was harvested $[13,14]$.

2.2. Auditory Function Tests. The ABR thresholds were measured using SmartEP (Intelligent Hearing System; Miami, FL, USA) [15] (Figure 2). The rats were anesthetized using $40 \mathrm{mg} / \mathrm{kg}$ Zoletil and $10 \mathrm{mg} / \mathrm{kg}$ xylazine. The electrodes were applied to the vertex (reference electrode), contralateral thigh (ground electrode), and ipsilateral retroauricular area (measuring electrode). The EC1 electrostatic speaker was coupled with a plastic earphone and inserted into the ipsilateral external auditory canal. Tone bursts of 4, 8, 16, and $32 \mathrm{kHz}$ (duration: $1562 \mu \mathrm{s}$, envelope: Blackman, stimulation rate: $21.1 / \mathrm{s}$, amplitude: $90-20 \mathrm{~dB}$ SPL) were applied. The ABRs were averaged for 1024 sweeps. The lowest sound amplitude with wave III was defined as the auditory threshold.
2.3. Histologic Examination of the Cochlea and Hippocampus. Hematoxylin and eosin (H\&E) staining and cochlear whole mount immunofluorescence were conducted for histologic examination of cochlea. Cochleae of control and noise group rats were immersion-fixed with $4 \%$ paraformaldehyde. Using $120 \mathrm{Mm}$ ethylenediaminetetraacetic acid, the bony labyrinth was decalcified for 5 days. For H\&E staining, the decalcified cochleae were dehydrated and embedded in paraffin. Then, $10 \mu \mathrm{m}$ sections were cut on a rotary microtome and mounted on glass slides. To examine the outer hair cells, cochlear whole mount examinations were conducted. The decalcified cochleae were dissected for the membranous labyrinth, and then, cochlear outer hair cells were identified. After washing the slides, blocking (1\% Triton X-100, 1\% bovine serum albumin (BSA) diluted in $10 \mathrm{mM}$ PBS $(\mathrm{pH} 7.4))$ and permeabilization $(0.1 \%$ Triton $\mathrm{X}-100,1 \%$ bovine serum albumin (BSA) diluted in $10 \mathrm{mM}$ PBS ( $\mathrm{pH}$ 7.4)) were performed for 1 hour at room temperature. After removing blocking solution, anti-myosin 7A (Santa Cruz, sc74516, Oregon, USA) was treated overnight at $4^{\circ} \mathrm{C}$. After removing primary antibody solution, secondary antibody (Alexa 594, Abcam, ab150108, Cambridge, UK) solution $(0.1 \%$ Triton X-100, $1 \%$ BSA) was incubated for 3 hours at room temperature. After removing secondary antibody solution, the tissue slides were dipped in $4^{\prime}$-6-diamidino-2-phenylindole dihydrochloride for 1 hour. The stained tissue slides were imaged using a confocal microscope (Zeiss LSM 880, Zeiss, Oberkochen, Land BadenWurttemberg, Germany).

For hippocampal histologic examination, whole brains of control and noise group rats were immersion-fixed in $4 \%(v / v)$ paraformaldehyde. The specimens were dehydrated and embedded in paraffin with optimal cutting temperature solution. For histological examination, $10 \mu \mathrm{m}$ sections were cut on a rotary microtome and mounted on glass slides. $\mathrm{H} \& \mathrm{E}$ staining of the hippocampus was performed to evaluate the histology of the hippocampus, especially the cornu ammonis (CA3) region. Each slide was dipped in xylene for $10 \mathrm{~min}$ to remove paraffin, followed by sequential washes with $100,95,90,80$, and $70 \%$ ethanol for $10,10,5,3$, and $1 \mathrm{~min}$, respectively. After washing in distilled water, the sections were stained with hematoxylin for $10 \mathrm{~min}$ and eosin for $3 \mathrm{~min}$. The stained tissue slides were imaged using an EVOSTMXL Core Imaging System (Invitrogen, Carlsbad, CA, USA, \#AMEX1000). To visualize the neurons of the hippocampus, Nissl staining was performed. Briefly, deparaffinized brain sections were permeabilized and stained with NeuroTrace 530/615 Red Fluorescent Nissl (N21482, Thermo Fisher Scientific) for $20 \mathrm{~min}$. After PBS washes, DAPI containing mounting solution was used for mounting. Images were photographed by using a TCS SP5II confocal microscope (Leica, Wetzlar, Germany).

2.4. miRNA Microarray Analyses. A total of 12 hippocampal tissues (six rats per group) were used for microarray analysis. RNA preparation and microarray analyses were conducted by BioCoRE ${ }^{\circledR}$. The tissue was lysed using the TRIzol reagent (Life Technologies, USA). The purified RNA was quantified using a NanoDrop 1000 


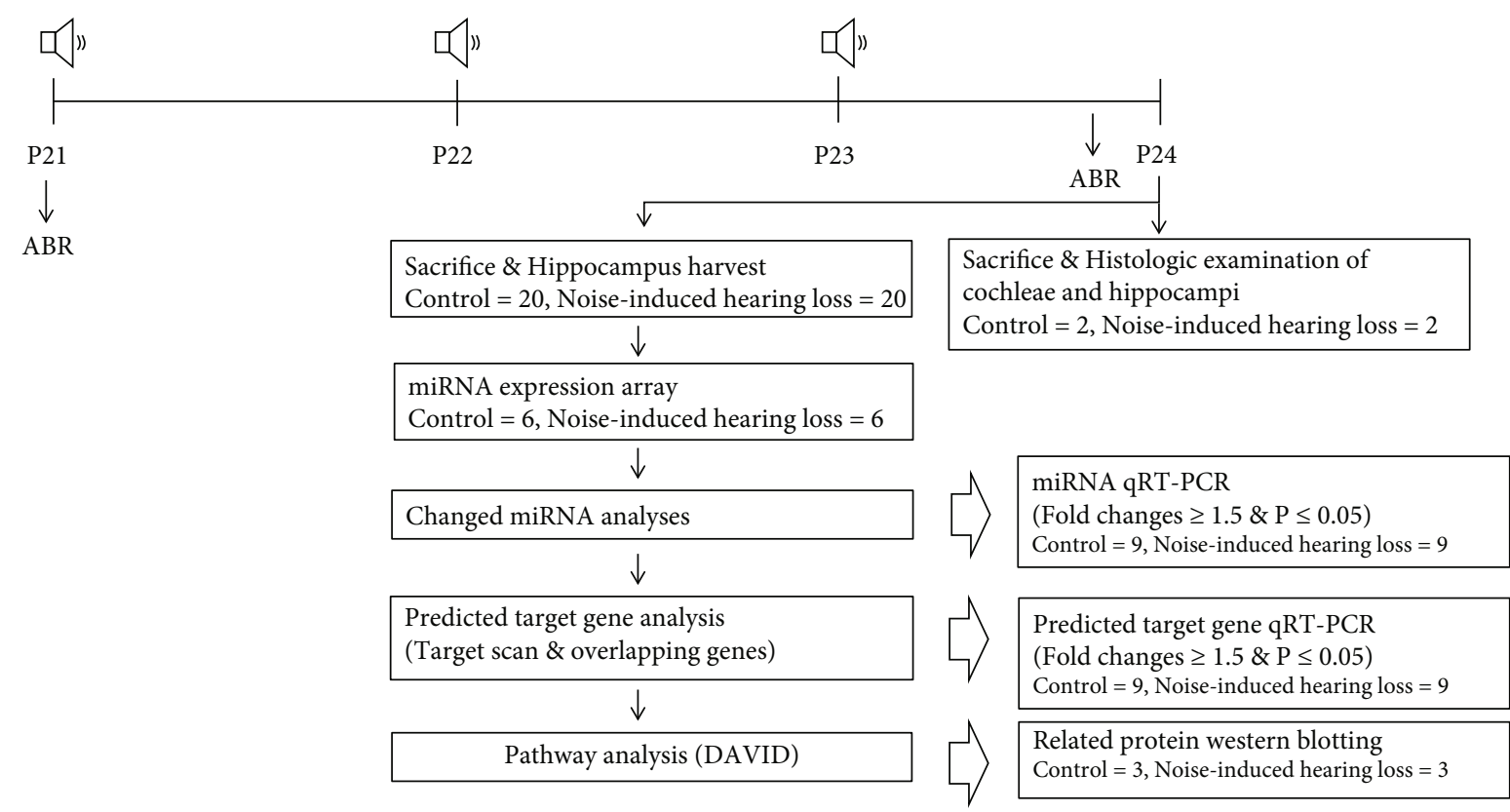

FIGURE 1: The miRNA microarray in the noise-induced hearing loss rats ( $n=20$ per group). Noise was exposed for three days, and auditory threshold shifts were checked using auditory brainstem response (ABR) threshold measures. The histologic examination of cochleae and hippocampi was conducted ( $n=2$ per group). The miRNA microarray was conducted using hippocampi of the noise-induced hearing loss and control rats ( $n=6$ per group). The predicted target gene analyses were performed, and their expression levels were estimated using qRT-PCR ( $n=9$ per group). The related proteins of identified pathways were evaluated using western blotting ( $n=3$ per group).

spectrophotometer (Thermo Scientific; Madison, WI, USA), and their quality was checked using an Agilent 2100 BioAnalyzer (Agilent Technologies; Santa Clara, CA, USA). Then, 500 ng RNA was labeled using the FlashTag Biotin RNA Labeling Kit (Affymetrix Inc.; Santa Clara, CA, USA) and hybridized using GeneChip miRNA 4.0 microarrays (Affymetrix Inc.), which contains 30,434 total mature miRNA probe sets, including 728 rat mature miRNA probe sets and 2578 human probe sets (data sheet: GeneChip miRNA 4.0 and Affymetrix miRNA 4.1 Arrays.pdf). Affymetrix miRNA arrays contain all miRNAs in miRBase Release 20. The arrays were scanned with the Affymetrix GeneChip Scanner 3000 and stored as CEL files. Raw analysis was conducted using the Transcriptome Analysis Console ${ }^{\mathrm{TM}}$ (TAC) software. CEL files were introduced into the Gene Expression Workflow in GeneSpring GX version 14.9.1 (Agilent Technologies Inc.). The RMA algorithm (background correction, $\log 2$ transformation, and probe set summarization) was implemented using default settings in GeneSpring software. Principal component analysis was performed using a covariance dispersion matrix as part of the quality control of the data. The miRNAs with $\geq 1.5$ fold-change in expression between the noise and control group rats were considered differentially expressed. A heat map (TreeView ver.1.1.6r4) was presented for the $\log 2$ transformed differentially expressed miRNAs.

2.5. Confirmation of miRNA Expression Using qRT-PCR. Nine rats from each group were used for qRT-PCR analysis. Using a miScript ${ }^{\circledR}$ II RT kit (Qiagen; Hilden, Germany), $2 \mu \mathrm{g}$ RNA was mixed with reverse transcription master mix and incubated for $60 \mathrm{~min}$ at $37^{\circ} \mathrm{C}$, after which the mixture was incubated for $5 \mathrm{~min}$ at $95^{\circ} \mathrm{C}$ and then placed on ice. A total of $20 \mu \mathrm{L} \mathrm{cDNA}$ was diluted to $1: 16$ and used as the template cDNA. The miScript SYBR ${ }^{\circledR}$ Green PCR kit (Qiagen) was used with miScript Primer Assay reagents (Qiagen) for qRT-PCR. U6 small nuclear RNA was used as the endogenous control. The miScript Primer Assay reagents and the reaction mix were dispensed into wells containing template cDNA, sealed with a film, and centrifuged at $1000 \times g$ for $1 \mathrm{~min}$ at room temperature. The PCR conditions were as follows: initial activation for $15 \mathrm{~min}$ at $95^{\circ} \mathrm{C}$, followed by 40 cycles of denaturation, annealing, and extension. The fluorescence data were collected during the extension phase. The reactions were performed using an ABI 7500 real-time PCR system (Applied Biosystems; Foster City, CA, USA). Relative quantification values were obtained for each target gene using the observed cycle threshold $(\mathrm{Ct})$ results and the $2^{-\Delta \Delta \mathrm{Ct}}$ method.

2.6. The Target Gene Predictions for miRNA. The predicted target genes were retrieved for each differentially expressed miRNA using TargetScan (http://www.targetscan.org/vert $72 /$ ), and the overlapping predicted target genes were identified. The mRNA expression of predicted target genes of five or more miRNAs was explored using qRT-PCR $[16,17]$. The ViiA7 real-time PCR system (Applied Biosystems; Carlsbad,

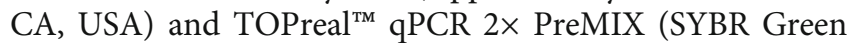
with low ROX; Enzynomics; Daejeon, Korea) were used. The primer sets used are described in Table 1. All primers were tested for amplification efficiency. Glyceraldehyde 3phosphate dehydrogenase was used as a reference gene to normalize the amplicon levels using the $2^{-\Delta \Delta \mathrm{Ct}}$ method. Then, the expression fold changes of each group were 

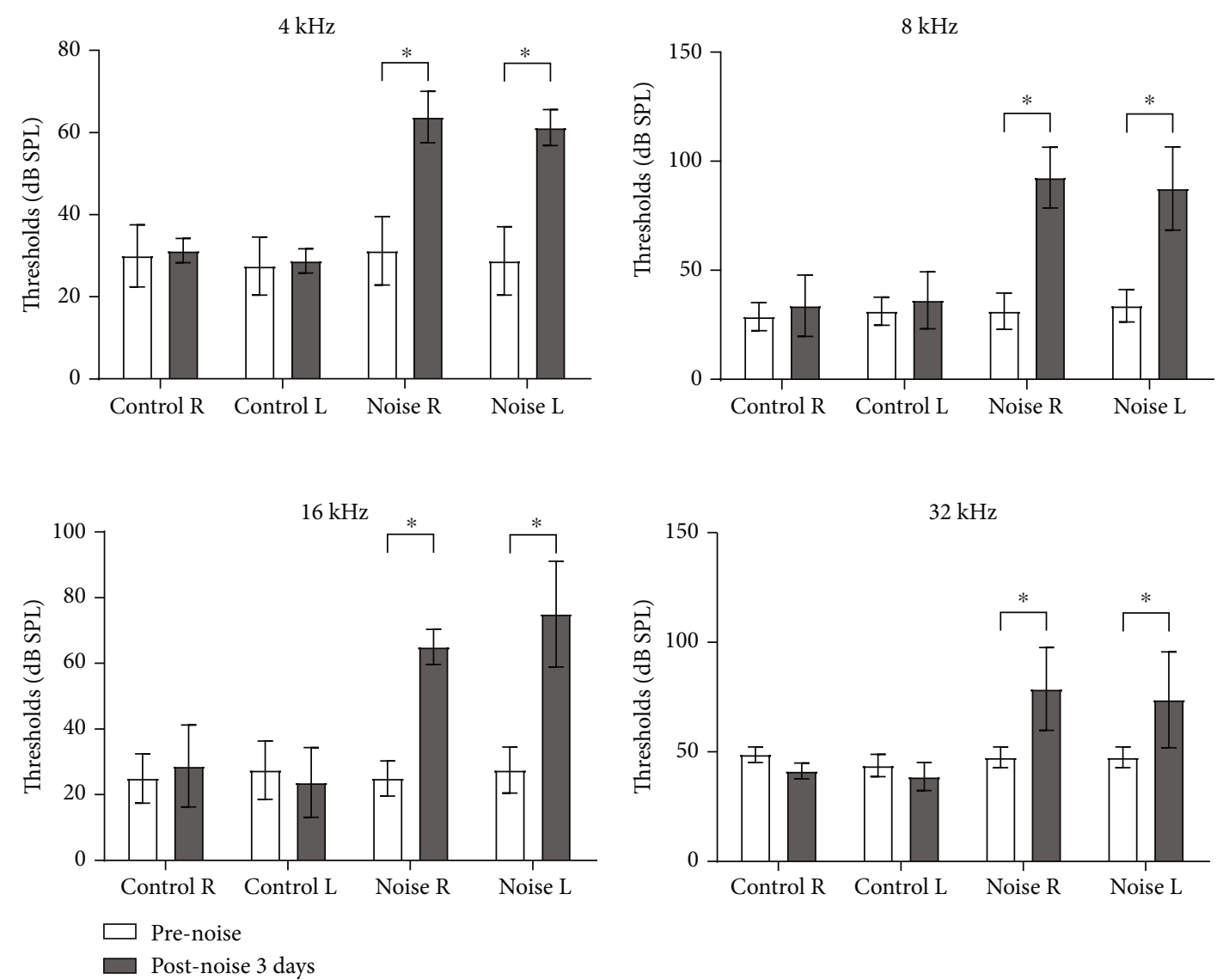

FIgURE 2: The auditory threshold shifts after noise exposure. The auditory brainstem response (ABR) thresholds were increased after noise exposure (postnoise 3 days) compared to those before noise exposure (prenoise) at $4,8,16$, and $32 \mathrm{kHz}\left({ }^{*} P<0.05\right.$, paired $t$-test between prenoise and postnoise 3 days; R: right side of the ear; L: left side of the ear).

calculated and compared to those of the control group. Based on the predicted target genes confirmed using qRT-PCR, the pathways were further analyzed using DAVID Bioinformatics Resources 6.8 (https://david.ncifcrf .gov/tools.jsp).

2.7. Western Blotting. The protein levels of megalin, angiotensin-converting enzyme 2 (ACE2), and tumor necrosis factor $\alpha(\mathrm{TNF} \alpha)$ were measured using western blotting. The hippocampal tissue was lysed using lysis buffer (Preprep, Intron). The protein was purified and quantified using a microplate reader. The quantified protein samples were loaded to $8-10 \%$ sodium dodecyl sulfate-polyacrylamide gel electrophoresis. The gels were transferred to polyvinylidene difluoride membranes (Merck Millipore, Burlington, MA, USA) and incubated in blocking buffer (5\% nonfat dry milk in Tris-buffered saline containing Tween-20) for 1 hour. The membranes were incubated with 1:1000 of anti-megalin (Santa Cruz, Dallas, Texas, U.S.A., Sc515772), anti-ACE2 (Santa Cruz, Dallas, Texas, U.S.A., Sc390851), anti-TNF $\alpha$ (Abcam, Cambridge, England, Ab6671), and anti-rabbit monoclonal $\beta$-actin (Cell Signaling Technology, Danvers, MA, USA, D6A8). Then, membranes were incubated with horseradish peroxidase- (HRP-) conjugated secondary antibodies (anti-rabbit IgG, HRP-linked (Cell Signaling Tech- nology, \#7074S) and goat anti-mouse IgG H\&L (HRP) (Abcam, Cambridge, England, \#ab97023)). Protein bands were evaluated using an enhanced chemiluminescence kit (Bio-Rad, Hercules, CA, USA) and quantified using ImageJ software (National Institutes of Health, Bethesda, MD, USA).

2.8. Statistical Analysis. The paired $t$-test was used to compare the ABR thresholds at pre- and postnoise exposures. The Mann-Whitney $U$ test was used for analyzing the mRNA and miRNA expression levels in the noise and control group rats, after conducting the Shapiro-Wilk test. The graphs are presented as mean \pm standard deviation (SD). SPSS software (ver. 21.0; IBM Corp.; Armonk, NY, USA) was used. $P \leq 0.05$ was considered statistically significant.

\section{Results}

Auditory threshold shifts were observed at $4,8,16$, and $32 \mathrm{kHz}$ following noise exposure (Figure 2). The auditory thresholds were higher in the noise group 3 days after noise exposure than those before noise exposure (all $P<0.05$ ).

Compared to the control group, the cochleae of white group rats showed loss and disorientations of outer hair cells and spiral ganglial cells (Figure 3). 
TABLE 1: Oligonucleotide primer sequences for quantitative reverse transcriptase polymerase chain reaction.

\begin{tabular}{|c|c|c|c|c|c|}
\hline Gene & Primer sequence (forward) & Primer sequence (reverse) & $\begin{array}{c}\text { Annealing } \\
\text { temperature }\left({ }^{\circ} \mathrm{C}\right)\end{array}$ & $\begin{array}{l}\text { Product size } \\
\text { (bp) }\end{array}$ & $\begin{array}{l}\text { Sequence } \\
\text { number }\end{array}$ \\
\hline Bend4 & $\begin{array}{c}5^{\prime} \text {-CTTTCTTGTTCAGCCGCCAG- } \\
3^{\prime}\end{array}$ & $\begin{array}{c}5^{\prime} \text {-CCCCCACCTTCCGAAA } \\
\text { AAGA-3' }\end{array}$ & 60 & 108 & $\mathrm{NM}_{020088.1}$ \\
\hline Creb1 & $\begin{array}{c}5^{\prime} \text {-TCC CAC TGT AAC CTT AGT } \\
\text { GCA G-3' }\end{array}$ & $\begin{array}{c}5^{\prime} \text {-ATA ACT GAT GGC TGG GCC } \\
\text { G-3 }\end{array}$ & 60 & 84 & $\mathrm{NM}_{134}$ \\
\hline Creb5 & $\begin{array}{c}5^{\prime} \text {-CATCTCTCGAGACCCGCTAC- } \\
3^{\prime}\end{array}$ & $\begin{array}{c}5^{\prime} \text {-TGGTCCTCTGTTGGGA } \\
\text { AACG- } 3^{\prime}\end{array}$ & 60 & 124 & $\mathrm{NM}_{001134621.1}$ \\
\hline Adcy6 & $\begin{array}{c}5^{\prime} \text {-TACATGAGCTGCCTCAAGA } \\
\text { ATGT-3' }\end{array}$ & $\begin{array}{c}5^{\prime} \text {-GTGTGGTCACCTCAGTGTC } \\
\text { AT- } 3^{\prime}\end{array}$ & 60 & 169 & $\mathrm{NM}_{012821.4}$ \\
\hline Kcnj9 & $\begin{array}{c}5^{\prime} \text {-AGCTGGCAGAAATGAAGAG } \\
\text { GA- } 3^{\prime}\end{array}$ & $\begin{array}{c}5^{\prime} \text {-ACACACTCAGTCCATTGGG } \\
\text { TT- } 3^{\prime}\end{array}$ & 60 & 138 & $\mathrm{NM}_{017297.2}$ \\
\hline Pten & $\begin{array}{c}5^{\prime} \text {-TATCTTGTGCTCACCCTGA } \\
\text { CAAA- } 3^{\prime}\end{array}$ & $\begin{array}{c}5^{\prime} \text {-AGAAGTATCGGTTGGCCTT } \\
\text { GT- } 3^{\prime}\end{array}$ & 60 & 70 & $\mathrm{NM}_{031606.1}$ \\
\hline Rps6ka5 & $\begin{array}{c}5^{\prime} \text {-TCCCAAGAAGCGATTGGGA } \\
\text { T- } 3^{\prime}\end{array}$ & $\begin{array}{c}5^{\prime} \text {-CGTCCCGGATCACTGG } \\
\text { TTTA- }{ }^{\prime}\end{array}$ & 60 & 83 & $\begin{array}{c}\mathrm{NM}_{-} \\
001108048.1\end{array}$ \\
\hline Nfatc3 & $\begin{array}{c}5^{\prime} \text {-TAACCTCCACCCATTTGCCA- } \\
3^{\prime}\end{array}$ & $\begin{array}{c}5^{\prime} \text {-GACCGAAGATGGTATTATG } \\
\text { TGCTG-3' }\end{array}$ & 60 & 73 & $\begin{array}{c}\mathrm{NM}_{-} \\
001108447.1\end{array}$ \\
\hline Rictor & $\begin{array}{c}5^{\prime} \text {-AATACCCTGCAGCGATCCTC- } \\
3^{\prime}\end{array}$ & $\begin{array}{c}5^{\prime} \text {-GGAGGCACATGCTTTG } \\
\text { ACTG- } 3^{\prime}\end{array}$ & 60 & 155 & $\begin{array}{c}\mathrm{NM}_{-} \\
008775080.2\end{array}$ \\
\hline Spred 1 & $\begin{array}{c}5^{\prime} \text {-CCCGTTCCCTGGTGAAAGAT- } \\
3^{\prime}\end{array}$ & $\begin{array}{c}5^{\prime} \text {-GGTATCTGGCTCACTTGGC } \\
\text { T- } 3^{\prime}\end{array}$ & 60 & 145 & 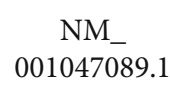 \\
\hline Cdh4 & $\begin{array}{c}5^{\prime} \text {-GCTGGTAGCCCAGACATCA } \\
\text { T- } 3^{\prime}\end{array}$ & $\begin{array}{c}5^{\prime} \text {-TCTGGTGTTGTTGAGGCCA } \\
\text { T- } 3^{\prime}\end{array}$ & 60 & 122 & $\frac{\mathrm{NM}_{-}}{017602710.1}$ \\
\hline Cdh6 & $\begin{array}{c}5^{\prime} \text {-GTTACAACGACGAAGGTGG } \\
\text { C- } 3^{\prime}\end{array}$ & $\begin{array}{c}5^{\prime} \text {-TGCTGTCTCCATGGCT } \\
\text { TAGG-3' }\end{array}$ & 60 & 85 & $\mathrm{NM}_{0127.1}$ \\
\hline$D v l 3$ & $\begin{array}{c}5^{\prime} \text {-CCTTCAATGGAACGCA } \\
\text { CAGG-3' }\end{array}$ & $\begin{array}{c}5^{\prime} \text {-CGTTGGGCAGATACCA } \\
\text { AGGA-3' }\end{array}$ & 60 & 128 & $\begin{array}{c}\mathrm{NM}_{-} \\
001107081.2\end{array}$ \\
\hline Pcyt1b & $\begin{array}{c}5^{\prime} \text {-GAGTAGCCGGATGCTA } \\
\text { CAGG-3' }\end{array}$ & $\begin{array}{c}5^{\prime} \text {-AGGTCTTGTTTGGGAG } \\
\text { CCAG-3' }\end{array}$ & 60 & 119 & $\mathrm{NM}_{173151.1}$ \\
\hline
\end{tabular}

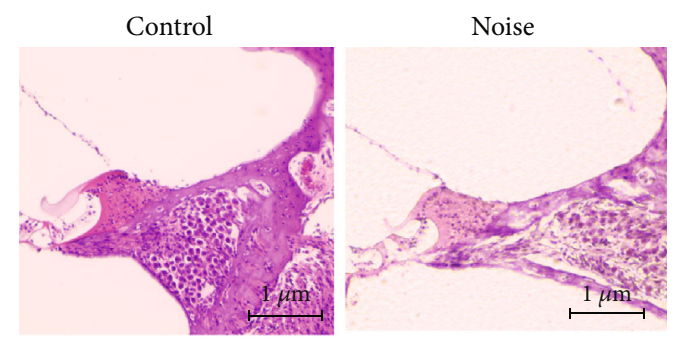

(a)
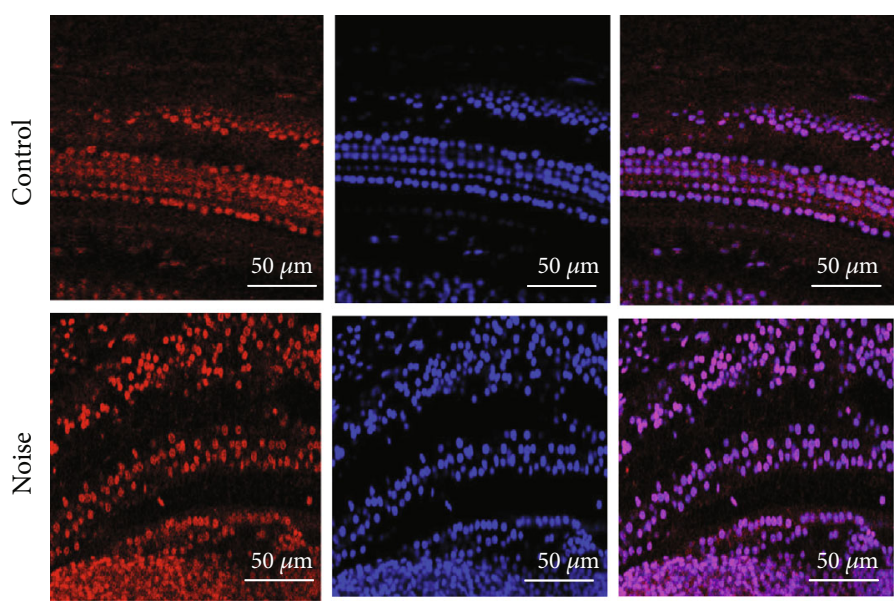

(b)

Figure 3: Comparison of cochlear histology between control and noise group rats. (a) Hematoxylin and eosin demonstrated loss of spiral ganglion cells in the noise group rats compared to the control group. (b) Cochlear whole mount immunofluorescence showed loss and disorientation of outer hair cells in the noise group rats. 
Gross morphology of hippocampi was not significantly different between the control and noise groups (Figure 4). However, the noise group showed pigmented and vacuolated cells in the CA3 region which was not obvious in the control group rats.

Nine miRNAs, miR-758-5p, miR-210-5p, miR-370-5p, miR-652-5p, miR-3544, miR-128-1-5p, miR-665, miR-188$5 \mathrm{p}$, and miR-874-5p, were upregulated in the hippocampal tissue of noise group rats (Table 2), which was confirmed using qRT-PCR. miR-758-5p was the most upregulated, with 2.23 -fold $(\mathrm{SD}=0.14)$ higher expression in noiseinduced rats than in normal rats.

The overlapping predicted target genes of five or more upregulated miRNAs were selected. qRT-PCR results demonstrated that Bend4, Creb1, Adcy6, Creb5, Kcnj9, and Pten were downregulated in the hippocampal tissues of the noise group rats (Figure 5).

The mRNA expression levels of Bend4, Creb1, Adcy6, Creb5, Kcnj9, and Pten were, respectively, 0.59-fold $(\mathrm{SD}=0.10, \quad P=0.001), \quad 0.52$-fold $(\mathrm{SD}=0.12, P<0.001)$, 0.59 -fold ( $\mathrm{SD}=0.10, P=0.03$ ), 0.20 -fold $(\mathrm{SD}=0.05, P<$ $0.001), \quad 0.47$-fold $(\mathrm{SD}=0.10, P=0.001)$, and 0.54 -fold $(\mathrm{SD}=0.12, P=0.004)$ lower in the noise group rats than in the control group rats. The pathway analysis suggested that these predicted target genes were related to the estrogen signaling pathway, vasopressin-regulated water reabsorption, thyroid hormone synthesis, aldosterone synthesis and secretion, insulin secretion, circadian entrainment, insulin resistance, cholinergic synapse, dopaminergic synapse, cGMPPKG signaling pathway, cAMP signaling pathway, PI3KAkt signaling pathway, TNF signaling pathway, and AMPK signaling pathway (Table 3 ).

In protein levels, the protein expression levels of megalin, ACE2, and TNF $\alpha$ were changed in the hippocampi of noise group rats, compared to those of control group rats (Figure 6). The protein expression level of megalin was 0.72 -fold lower in the noise group $(\mathrm{SD}=0.23, P=0.02)$. The protein expression level of ACE2 was 1.56-fold higher in the noise group $(\mathrm{SD}=0.26, P=0.004)$. The protein expression level of TNF $\alpha$ was 0.77 -fold lower in the noise group ( $\mathrm{SD}=0.18, P=0.037)$.

On the contrary, miR-448-3p, miR-204-5p, miR-204-3p, and miR-140-5p were downregulated in the noise group rats. Notably, qRT-PCR confirmed that miR-448-3p, miR204-5p, and miR-204-3p were downregulated, while miR$140-5 \mathrm{p}$ was upregulated $(1.63$-fold, $\mathrm{SD}=0.04)$. $\mathrm{miR}-448-3 \mathrm{p}$ was the most downregulated, with 0.18 -fold $(\mathrm{SD}=0.004)$ lower expression in noise-induced rats than in normal rats.

Rps6kas, Nfactc3, Rictor, Spred1, Cdh4, Cdh6, Dvl3, and $R c y t 1 b$ were the predicted target genes of the downregulated miRNAs (Table 2). qRT-PCR results demonstrated that these genes were upregulated in the noise group rats (Figure 7). The mRNA expression levels of Rps6kas, Nfactc3, Rictor, Spred1, Cdh4, Cdh6, Dvl3, and Rcyt1b were, respectively, 1.61-fold ( $\mathrm{SD}=0.24, P=0.019), 3.20$-fold $(\mathrm{SD}=0.64$ , $P=0.002), 3.53$-fold $(\mathrm{SD}=0.63, P=0.001), 1.33$-fold $(\mathrm{SD}=0.56, P=0.031), 3.05$-fold $(\mathrm{SD}=0.72, P=0.0008)$, 1.63 -fold ( $\mathrm{SD}=0.20, P=0.011), 1.65$-fold $(\mathrm{SD}=0.25, P=$ $0.015)$, and 1.86 -fold ( $S D=0.28, P=0.005)$ higher in the
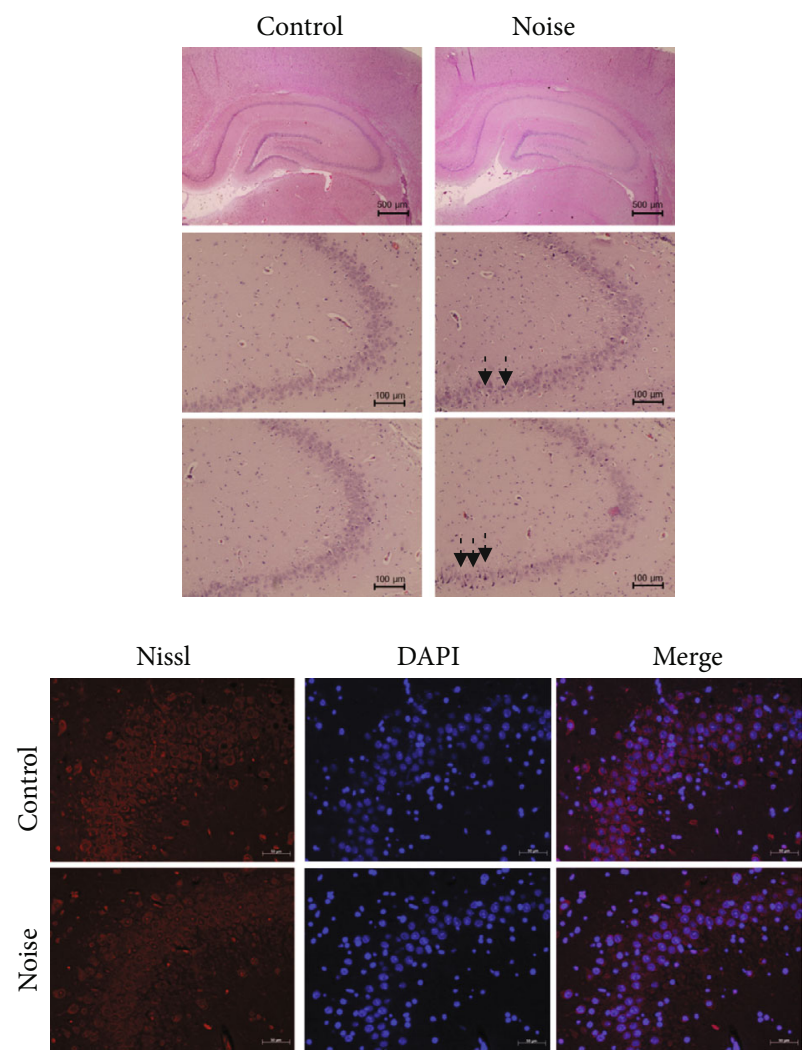

FIgURE 4: Comparison of hippocampal histology between control and noise group rats. Hematoxylin and eosin demonstrated pigmented (dotted arrows) and vacuolated (arrows) cells in the noise group rats compared to the control group. The Nissl staining of the CA3 region demonstrated neuronal cells of control and noise groups which did not show significant difference between groups.

noise group rats than in the control group rats. Pathway analysis for these upregulated genes suggested that $D v l 3$ and Nfactc3 are related to the Wnt signaling pathway.

\section{Discussion}

Noise-induced hearing loss changed the hippocampal expression levels of several miRNAs, including miR-7585p, miR210-5p, miR370-5p, miR-652-5p, miR-3544, miR128-1-5p, miR-665, miR-188-5p, miR-874-5p, miR-448-3p, miR-204-5p, miR-204-3p, and miR-140-5p. The predicted target genes of the upregulated miRNAs include Creb1, Adcy6, Creb5, Kcnj, and Pten, which are related to the estrogen signaling pathway, vasopressin-regulated water reabsorption, thyroid hormone synthesis, aldosterone synthesis and secretion, insulin secretion, circadian entrainment, insulin resistance, cholinergic synapse, dopaminergic synapse, cGMP-PKG signaling pathway, cAMP signaling pathway, PI3K-Akt signaling pathway, TNF signaling pathway, and AMPK signaling pathway. On the contrary, the predicted target genes of the downregulated miRNAs, such as miR448-3p, miR-204-5p, and miR-204-3p, include Rps6kas, Nfactc3, Rictor, Spred1, Cdh4, Cdh6, Dvl3, and Rcyt1b, which are related to the Wnt signaling pathway. These results 
TABLE 2: The differentially expressed miRNAs in noise-induced hearing loss rats and their predicted target genes.

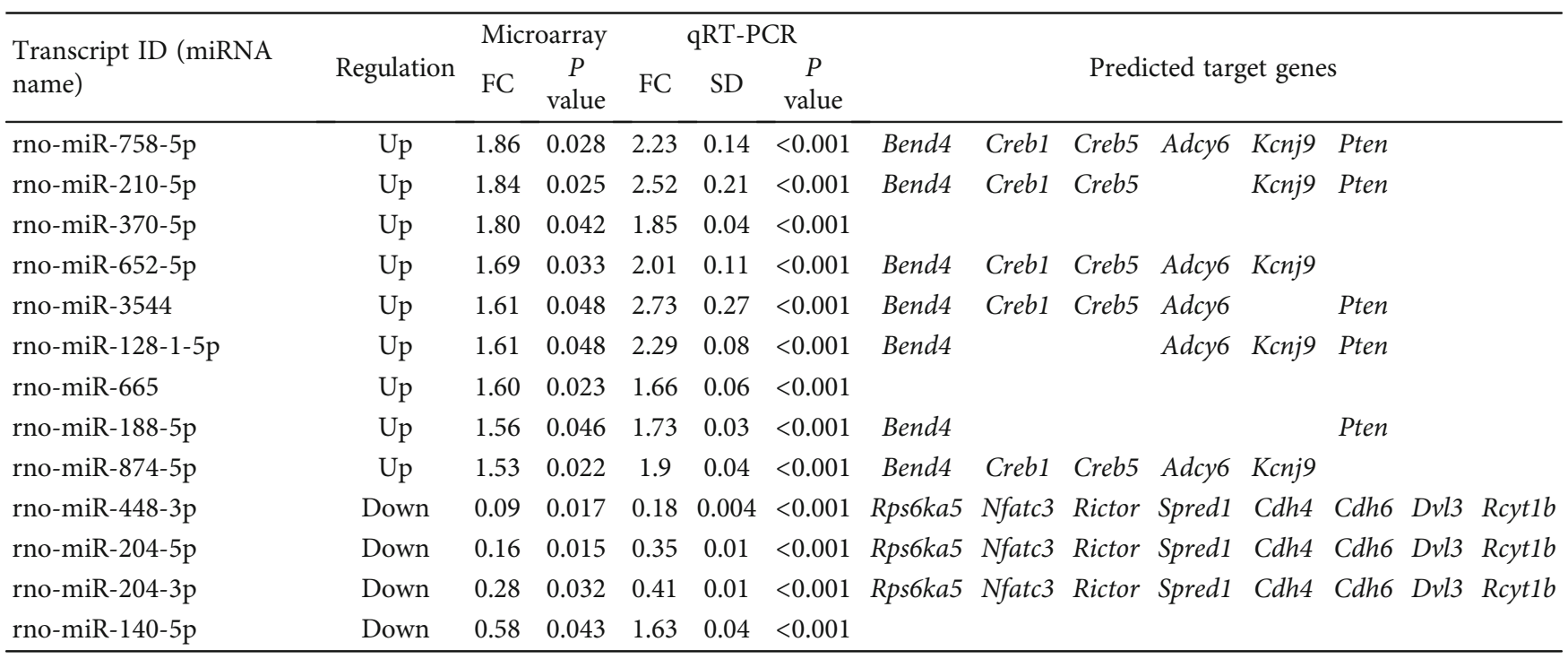
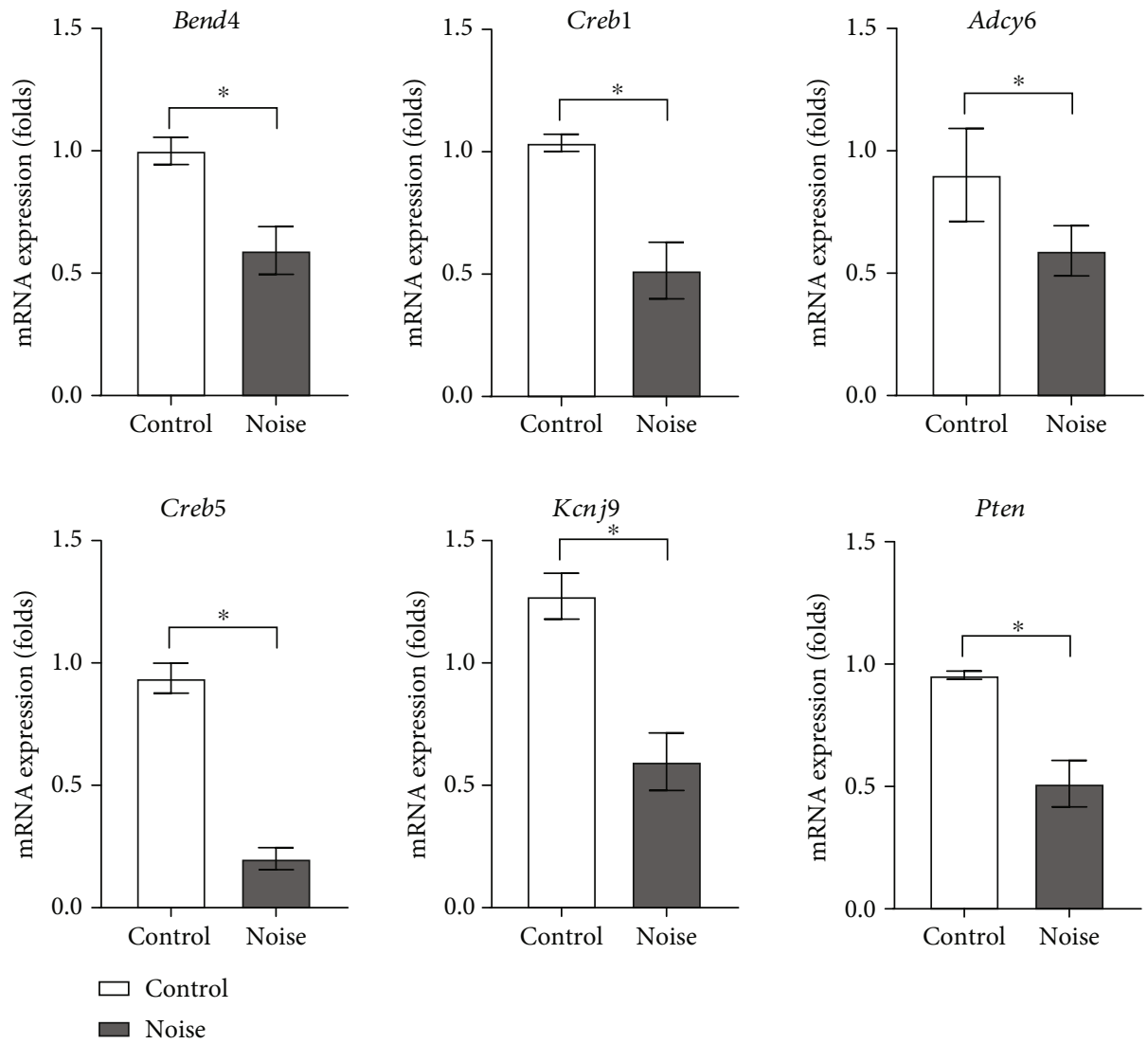

FIGURE 5: The predicted target genes of the upregulated miRNAs in the hippocampi of noise-induced hearing loss rats. The mRNA expression levels of Bend4, Creb1, Adcy6, Creb5, Kcnj9, and Pten were lower in the noise-induced hearing loss rats than in control rats.

suggest a link between noise-induced hearing loss and hippocampal changes, which could induce cognitive dysfunction.

Hippocampal changes due to hearing loss have been suggested in noise-induced as well as in age-related hearing loss rodent models [18]. Transient threshold shift (TTS) as well as permanent threshold shift (PTS) models $[19,20]$ showed biochemical, neuronal, and behavioral changes in the hippocampus after noise exposure [21-23]. Within $24 \mathrm{~h}$ after impulse noise exposure (peak sound pressure: $165 \mathrm{~dB}$ SPL 
TABLE 3: Pathways related to the predicted target genes of differentially expressed miRNAs in noise-induced hearing loss rats.

\begin{tabular}{|c|c|c|c|c|c|c|c|c|}
\hline Term & Regulations & $P$ value & Benjamini & & & Genes & & \\
\hline Estrogen signaling pathway & Down & $<0.001$ & 0.001 & Adcy6 & Creb1 & Creb5 & Kcnj9 & \\
\hline Vasopressin-regulated water reabsorption & Down & $<0.001$ & 0.006 & Adcy6 & Creb1 & Creb5 & & \\
\hline Thyroid hormone synthesis & Down & $<0.001$ & 0.009 & Adcy6 & Creb1 & Creb5 & & \\
\hline Aldosterone synthesis and secretion & Down & 0.001 & 0.009 & Adcy6 & Creb1 & Creb5 & & \\
\hline Insulin secretion & Down & 0.001 & 0.009 & Adcy6 & Creb1 & Creb5 & & \\
\hline Circadian entrainment & Down & 0.001 & 0.009 & Adcy6 & Creb1 & & Kcnj9 & \\
\hline Insulin resistance & Down & 0.001 & 0.009 & & Creb1 & Creb5 & & Pten \\
\hline Cholinergic synapse & Down & 0.001 & 0.009 & & Creb1 & Creb5 & Kcnj9 & \\
\hline Dopaminergic synapse & Down & 0.002 & 0.011 & & Creb1 & Creb5 & Kcnj9 & \\
\hline cGMP-PKG signaling pathway & Down & 0.003 & 0.013 & Adcy6 & Creb1 & Creb5 & & \\
\hline cAMP signaling pathway & Down & 0.004 & 0.018 & Adcy6 & Creb1 & Creb5 & & \\
\hline PI3K-Akt signaling pathway & Down & 0.011 & 0.050 & & Creb1 & Creb5 & & Pten \\
\hline TNF signaling pathway & Down & 0.055 & 0.170 & & Creb1 & Creb5 & & \\
\hline AMPK signaling pathway & Down & 0.064 & 0.190 & & Creb1 & Creb5 & & \\
\hline Wnt signaling pathway & Up & 0.071 & 1.000 & $D v l 3$ & Nfatc3 & & & \\
\hline
\end{tabular}
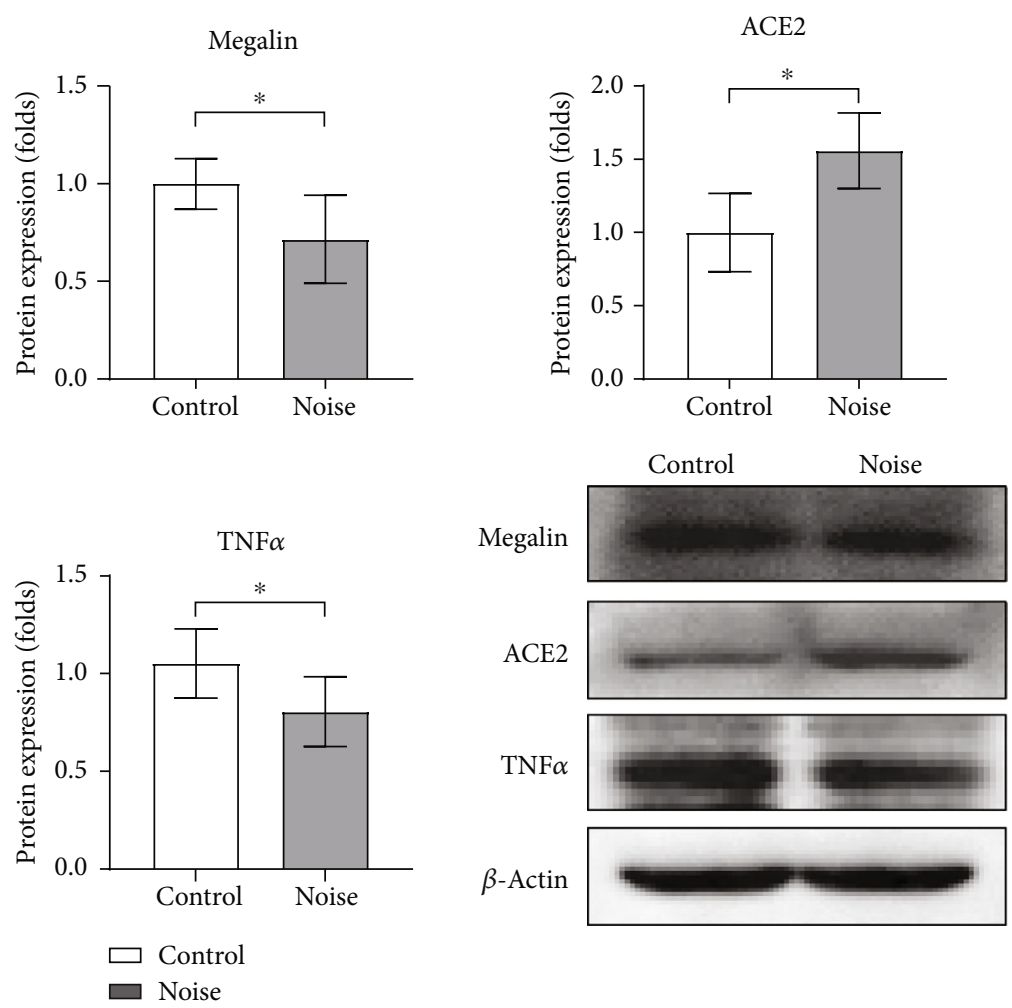

FIGURE 6: The protein expression levels of megalin, ACE2, and TNF $\alpha$ in the hippocampi of noise and control group rats. The protein expression levels of megalin and $\mathrm{TNF} \alpha$ were lower, and those of ACE2 were higher in the noise group rats than in control group rats $\left({ }^{*} P<0.05\right)$.

for $100 \mathrm{~ms}$ ), glutamate-N-methyl-D-aspartic acid receptor (NMDAR) signaling was activated, tau hyperphosphorylation increased, and the Morris water maze test indicated diminished spatial memory [23]. The number of hippocampal neurons decreased within 2-6h after noise exposure ( $4 \mathrm{kHz}, 104 \mathrm{~dB}$ SPL for $30 \mathrm{~min})$ [21]. The long-term potentiation of the hippocampus was inhibited after exposure to
$110 \mathrm{~dB}$ SPL noise $(12 \mathrm{kHz})$ for one minute [22]. Stress responses, including oxidative stress, inflammation, hormonal changes, and neuronal or synaptic changes related to auditory signaling deprivation, may induce hippocampal dysregulations [18]. The stress hormones, such as norepinephrine and serotonin, were elevated in the hippocampus following noise exposures [24]. In addition, inflammatory 

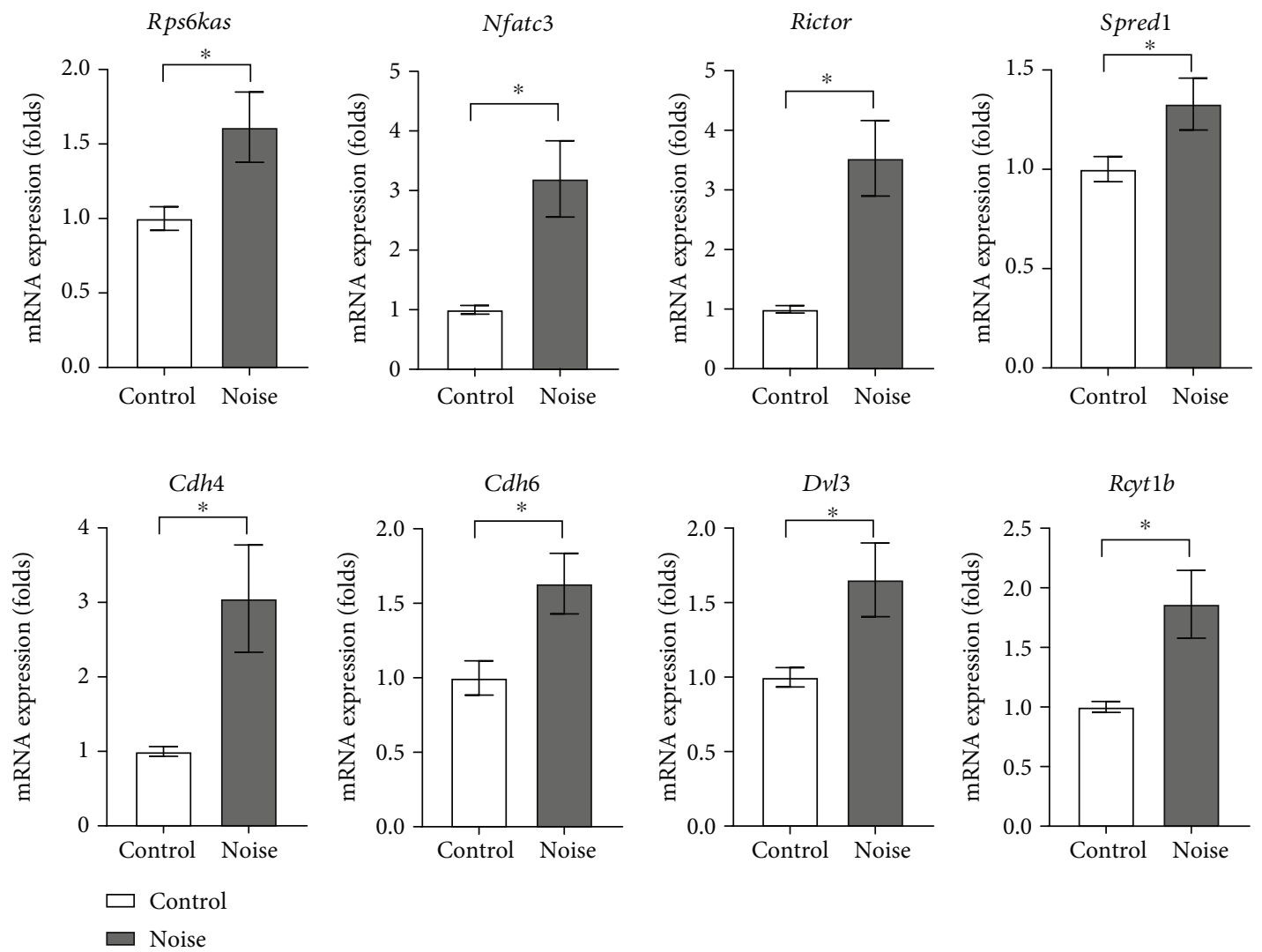

FIgURE 7: The predicted target genes of the downregulated miRNAs in the hippocampi of noise-induced hearing loss rats. The mRNA expression levels of Rps6ksa, Nfactc3, Rictor, Spred1, Cdh4, Cdh6, Dvl3, and Rcyt1b were higher in the noise-induced hearing loss rats than in control rats.

changes with dystrophic microglia and diminished neurogenesis were noted in hippocampi of noise-induced hearing loss mice [25]. The results of this study also predicted the changes in signaling pathways related to hormones, such as estrogen signaling pathway, vasopressin-regulated water reabsorption, thyroid hormone synthesis, aldosterone synthesis and secretion, insulin secretion, circadian entrainment, insulin resistance, and inflammation, such as the cGMP-PKG signaling pathway, cAMP signaling pathway, PI3K-Akt signaling pathway, TNF signaling pathway, and AMPK signaling pathway, and synaptic changes, such as cholinergic synapses and dopaminergic synapses in the hippocampi of the noise group rats. To support this, the protein expression levels of estrogen receptor protein of megalin and $\mathrm{TNF} \alpha$ were lower in the noise group [26]. Megalin is known as a low-density lipoprotein receptor-related protein family and was suggested to have a critical role in hearing by interacting with estrogen [26]. In addition, the expression of ACE2 was higher in the noise group than in the control group in the present study. The balance between ACE and ACE2 is critical to regulate the level of angiotensin II [27]. These hippocampal changes after noise exposure could be induced by the miRNA changes following noise exposure.

Little is known about miRNA changes in the hippocampus after noise exposure. Notably, a recent study reported miRNA changes in the hippocampus of a surgically deafened $\mathrm{AD}$ rat model and demonstrated that miR-376a-3p and
miR-598-3p expression changes in the hippocampal tissue of a rat model of amyloid- $\beta$ infusion and bilateral cochlear ablation [10]. However, no relevant target gene or pathway was identified in the previous study. In this study, miR758-5p, miR210-5p, miR370-5p, miR-652-5p, miR-3544, miR-128-1-5p, miR-665, miR-188-5p, and miR-874-5p expression increased in the hippocampi of the noise group rats. Among these miRNAs, miR-188-5p regulates synaptic plasticity as its expression level increases with long-term potentiation [28]. In addition, miR-188-5p diminishes amyloid- $\beta$ peptide $_{1-42}$-mediated synapse elimination and dysfunction and cognitive deficits by downregulating neuropilin-2 (Nrp-2) in a 5XFAD mouse model of AD [29]. Nrp-2 is a receptor for semaphoring $3 \mathrm{~F}$, which plays a role in synaptic plasticity and axon guidance [30]. Thus, miR-188-5p upregulation in the hippocampal tissue of noise-induced rats may imply the induction of synaptic changes in the hippocampus after hearing loss. Moreover, miR-210-5p, which was found to be upregulated in this study, was also reported to increase in a rat model of vascular dementia [31]. miR-210-5p increase induced loss of synapses in the CA 1 region of the hippocampus in vascular dementia rats and suppressed the mRNA expression of synaptosomal-associated protein 25 [31].

In contrast, miR-448-3p, miR-204-5p, miR-204-3p, and miR-140-5p expression decreased in the hippocampi of the noise group rats in this study. miR-204-3p was 
downregulated in the APPswe/PS1De9 (APP/PS) mouse model of AD [32]. miR-204-3p replenishment ameliorated memory deficits and reduced oxidative stress in APP/PS1 mice by suppressing nicotinamide adenine dinucleotide phosphate oxidase 4 [32]. Although very few studies have focused on other downregulated miRNAs, the predicted target gene and pathway analyses suggested the relationship with the Wnt signaling pathway, which is involved in multiple processes involved in neurogenesis and synaptic plasticity [33].

This study explored the miRNA changes in rat hippocampi one day after noise exposure. This time frame was chosen based on previous studies reporting hippocampal changes within one day after noise exposure $[21,23]$. The temporal and regional changes in miRNAs warrant further study. In addition, although several predicted target genes and pathways related to the changed miRNAs were explored, the plausible molecular networks need to be elucidated through rescue studies using these miRNAs.

\section{Conclusions}

miR-758-5p, miR210-5p, miR370-5p, miR-652-5p, miR3544, miR-128-1-5p, miR-665, miR-188-5p, and miR-874$5 p$ were upregulated, whereas miR-448-3p, miR-204-5p, miR-204-3p, and miR-204-3p were downregulated in the hippocampi of rats with noise-induced hearing loss. These miRNAs mediate hormonal responses, inflammation, and synaptic changes.

\section{Data Availability}

The raw data of experiments used to support the findings of this study are available from the corresponding author upon request.

\section{Disclosure}

The funders had no role in the design of the study, in the collection, analyses, or interpretation of data, in the writing of the manuscript, or in the decision to publish the results.

\section{Conflicts of Interest}

The authors declare no conflict of interest.

\section{Acknowledgments}

This research was supported by funding from the National Research Foundation (NRF) of Korea (NRF2018R1D1A1B07048092 and 2020R1A2C4002594).

\section{References}

[1] A. Shukla, M. Harper, E. Pedersen et al., "Hearing loss, loneliness, and social isolation: a systematic review," Otolaryngology and Head and Neck Surgery, vol. 162, no. 5, pp. 622-633, 2020.

[2] C. Coyat, C. Cazevieille, V. Baudoux, P. Larroze-Chicot, B. Caumes, and S. Gonzalez-Gonzalez, "Morphological consequences of acoustic trauma on cochlear hair cells and the audi- tory nerve," The International Journal of Neuroscience, vol. 129 , no. 6 , pp. 580-587, 2019.

[3] A. L. Harrison Bush, J. J. Lister, F. R. Lin, J. Betz, and J. D. Edwards, "Peripheral hearing and cognition: evidence from the staying keen in later life (SKILL) study," Ear and Hearing, vol. 36, no. 4, pp. 395-407, 2015.

[4] C. H. Lee, K. W. Kim, S. M. Lee, and S. Y. Kim, "Effect of acute noise trauma on the gene expression profile of the hippocampus," BMC Neuroscience, vol. 21, no. 1, pp. 1-13, 2020.

[5] M. Chang, H. J. Kim, I. Mook-Jung, and S.-H. Oh, "Hearing loss as a risk factor for cognitive impairment and loss of synapses in the hippocampus," Behavioural Brain Research, vol. 372, article 112069, 2019.

[6] D. Su, W. Li, X. She et al., "Chronic noise exposure exacerbates AD-like neuropathology in SAMP8 mice in relation to Wnt signaling in the PFC and hippocampus," Scientific Reports, vol. 8, no. 1, pp. 1-10, 2018.

[7] S. L. Hollins and M. J. Cairns, "MicroRNA: small RNA mediators of the brains genomic response to environmental stress," Progress in Neurobiology, vol. 143, pp. 61-81, 2016.

[8] M. Esteller, "Non-coding RNAs in human disease," Nature Reviews Genetics, vol. 12, no. 12, pp. 861-874, 2011.

[9] F. Ye, S. Tian, H. Hu, and Z. Yu, "Electroacupuncture Reduces Scopolamine-Induced Amnesia via Mediating the miR210/SIN3A and miR-183/SIN3A Signaling Pathway," Molecular Medicine, vol. 26, no. 1, pp. 1-11, 2020.

[10] S. K. Mun, H. Chae, X. Y. Piao et al., "MicroRNAs related to cognitive impairment after hearing loss," Clinical and Experimental Otorhinolaryngology, vol. 14, no. 1, pp. 76-81, 2021.

[11] Y. Bouter, T. Kacprowski, F. Rößler, L. R. Jensen, A. W. Kuss, and T. A. Bayer, "miRNA alterations elicit pathways involved in memory decline and synaptic function in the hippocampus of aged Tg4-42 mice," Frontiers in Neuroscience, vol. 14, p. 951, 2020.

[12] S. Park, S. H. Han, B.-G. Kim et al., "Changes in microRNA expression in the cochlear nucleus and inferior colliculus after acute noise-induced hearing loss," International Journal of Molecular Sciences, vol. 21, no. 22, p. 8792, 2020.

[13] L. J. Kjonigsen, T. B. Leergaard, M. P. Witter, and J. G. Bjaalie, "Digital atlas of anatomical subdivisions and boundaries of the rat hippocampal region," Frontiers in Neuroinformatics, vol. 5, p. 2, 2011.

[14] S. Y. Kim, C. Min, B. Park, and H. G. Choi, "Bidirectional association between GERD and rheumatoid arthritis: two longitudinal follow-up studies using a national sample cohort," Clinical Rheumatology, vol. 40, no. 4, pp. 1249-1257, 2021.

[15] S. Y. Kim, H. Heo, D. H. Kim, H. J. Kim, and S. H. Oh, "Neural plastic changes in the subcortical auditory neural pathway after single-sided deafness in adult mice: a MEMRI study," Biomed Research International, vol. 2018, Article ID 8624745, 8 pages, 2018.

[16] S. Y. Kim, J. K. Kim, S. H. Park et al., "Effects of inhaled particulate matter on the central nervous system in mice," Neurotoxicology, vol. 67, pp. 169-177, 2018.

[17] S. A. Bustin, V. Benes, J. A. Garson et al., "The MIQE guidelines: minimum information for publication of quantitative real-time PCR experiments," Clinical Chemistry, vol. 55, no. 4, pp. 611-622, 2009.

[18] Y. Nadhimi and D. A. Llano, "Does hearing loss lead to dementia? A review of the literature," Hearing Research, vol. 402, article 108038, 2021. 
[19] M. Shukla, K. Roy, C. Kaur et al., "Attenuation of adverse effects of noise induced hearing loss on adult neurogenesis and memory in rats by intervention with adenosine $\mathrm{A}_{2 \mathrm{~A}}$ receptor agonist," Brain Research Bulletin, vol. 147, pp. 47-57, 2019.

[20] S. Y. Park, M. J. Kim, H. L. Kim, D. K. Kim, S. W. Yeo, and S. N. Park, "Cognitive decline and increased hippocampal ptau expression in mice with hearing loss," Behavioural Brain Research, vol. 342, pp. 19-26, 2018.

[21] T. J. Goble, A. R. Moller, and L. T. Thompson, "Acute highintensity sound exposure alters responses of place cells in hippocampus," Hearing Research, vol. 253, no. 1-2, pp. 52-59, 2009.

[22] J. L. de Deus, A. O. S. Cunha, A. L. Terzian et al., "A single episode of high intensity sound inhibits long-term potentiation in the hippocampus of rats," Scientific Reports, vol. 7, no. 1, p. 14094, 2017.

[23] B. Cui, M. Wu, X. She, and H. Liu, "Impulse noise exposure in rats causes cognitive deficits and changes in hippocampal neurotransmitter signaling and tau phosphorylation," Brain Research, vol. 1427, pp. 35-43, 2012.

[24] S. G. Jin, M. J. Kim, S. Y. Park, and S. N. Park, "Stress hormonal changes in the brain and plasma after acute noise exposure in mice," Auris, Nasus, Larynx, vol. 44, no. 3, pp. 272-276, 2017.

[25] H. Zhuang, J. Yang, Z. Huang et al., “Accelerated age-related decline in hippocampal neurogenesis in mice with noiseinduced hearing loss is associated with hippocampal microglial degeneration," Aging, vol. 12, no. 19, pp. 19493-19519, 2020.

[26] O. König, L. Rüttiger, M. Müller et al., "Estrogen and the inner ear: megalin knockout mice suffer progressive hearing loss," The FASEB Journal, vol. 22, no. 2, pp. 410-417, 2008.

[27] A. Kaparianos and E. Argyropoulou, "Local renin-angiotensin II systems, angiotensin-converting enzyme and its homologue ACE2: their potential role in the pathogenesis of chronic obstructive pulmonary diseases, pulmonary hypertension and acute respiratory distress syndrome," Current Medicinal Chemistry, vol. 18, no. 23, pp. 3506-3515, 2011.

[28] K. Lee, J. H. Kim, O. B. Kwon et al., "An activity-regulated microRNA, miR-188, controls dendritic plasticity and synaptic transmission by downregulating neuropilin-2," Journal of Neuroscience, vol. 32, no. 16, pp. 5678-5687, 2012.

[29] K. Lee, H. Kim, K. An et al., "Replenishment of microRNA188-5p restores the synaptic and cognitive deficits in 5XFAD mouse model of Alzheimer's disease," Scientific Reports, vol. 6, no. 1, p. 34433, 2016.

[30] H. Chen, A. Bagri, J. A. Zupicich et al., "Neuropilin-2 Regulates the Development of Select Cranial and Sensory Nerves and Hippocampal Mossy Fiber Projections," Neuron, vol. 25, no. 1, pp. 43-56, 2000.

[31] Z. Ren, J. Yu, Z. Wu et al., "MicroRNA-210-5p contributes to cognitive impairment in early vascular dementia rat model through targeting Snap25," Frontiers in Molecular Neuroscience, vol. 11, p. 388, 2018.

[32] W. Tao, L. Yu, S. Shu et al., "miR-204-3p/Nox4 mediates memory deficits in a mouse model of Alzheimer's disease," Molecular Therapy, vol. 29, no. 1, pp. 396-408, 2021.

[33] L. Jia, J. Pina-Crespo, and Y. Li, "Restoring Wnt/ $\beta$-catenin signaling is a promising therapeutic strategy for Alzheimer's disease," Molecular Brain, vol. 12, no. 1, p. 104, 2019. 